\title{
Nobiletin Exhibits Neuroprotective Effects against Mitochondrial Complex I Inhibition via Regulating Apoptotic Signaling
}

\author{
Khulan Amarsanaa ${ }^{1}$, Hye-Ji Kim ${ }^{1}$, Eun-A Ko ${ }^{1}$, Jaemin Jo $^{2}$ and Sung-Cherl Jung ${ }^{1,3,4 *}$ \\ ${ }^{1}$ Department of Physiology, School of Medicine, Jeju National University, ${ }^{2}$ Department of Internal Medicine, School of \\ Medicine, Jeju National University, ${ }^{3}$ Institute of Medical Science, Jeju National University, ${ }^{4}$ Interdisciplinary Graduate \\ Program in Advanced Convergence Technology \& Science, Jeju National University, Jeju 63243, Korea
}

\begin{abstract}
Nobiletin, a polymethoxylated flavonoid found in citrus, has been studied because of its modulatory functions in cellular signaling cascades, and effects to prevent mitochondrial calcium overload and neuronal cell death. Particularly, we previously reported that nobiletin induced changes in the mitochondrial membrane potential through $\mathrm{K}^{+}$channel regulation, suggesting that nobiletin might exert neuroprotective effects via regulating mitochondrial functions associated with the electron transport chain (ETC) system. This study investigated whether nobiletin regulated mitochondrial dysfunction mediated by ETC system downregulation by inhibiting complex I (CI) and complex III (CIII) in pure mitochondria and the cortical neurons of rats. The results showed that nobiletin significantly reduced mitochondrial reactive oxygen species (ROS) production, inhibited apoptotic signaling, enhanced ATP production and then restored neuronal viability under conditions of CI inhibition, but not CIII inhibition. These effects were attributed to the downregulation of translocation of apoptosis-induced factor (AIF), and the upregulation of CI activity and the expression of antioxidant enzymes such as Nrf2 and HO-1. Together with our previous study, these results indicate that the neuroprotective effects of nobiletin under mitochondrial dysfunction may be associated with its function to activate antioxidant signaling cascades. Our findings suggest the possibility that nobiletin has therapeutic potential in treating oxidative neurological and neurodegenerative diseases mediated by mitochondrial dysfunction.
\end{abstract}

Key words: Nobiletin, Mitochondria, Electron transport chains, Complex I, ROS, Apoptosis-inducing factor

\section{INTRODUCTION}

Nobiletin, known as 5,6,7,8,3','-hexamethoxyflavone, is a polymethoxylated flavonoid found in citrus, exerts multiple pharmacological activities in various diseases including neurological and cardiovascular diseases, metabolic disorders, and cancer, as well as inflammation and oxidative dysfunction [1]. Particularly, positive effects on the nervous system to improve cognitive function and

Submitted October 20, 2020, Revised December 10,2020,

Accepted December 25, 2020

* To whom correspondence should be addressed. TEL: 82-65-754-3834, FAX: 82-64-702-2687 e-mail: jungsc@jejunu.ac.kr effectively recover motor deficits under pathological conditions such as ischemia, Parkinson's and Alzheimer's diseases have been frequently reported [2-4]. In its cellular mechanisms, nobiletin regulates the $\mathrm{K}^{+}$influx and the potential $\left(\Delta \Psi_{\mathrm{m}}\right)$ of the mitochondrial inner membrane to prevent mitochondrial calcium overload and neuronal cell death and enhances metabolic rates by changing the mitochondrial matrix substrate $[5,6]$. These effects suggest that nobiletin may exhibit neuroprotective effects via regulating mitochondrial functions that are associated with the electron transport chain (ETC) system.

A number of previous studies have focused on the metabolic functions of mitochondria to determine the viability of different types of cells [7-11]. Based on the metabolic functions of mitochondria, the ETC system generates an electrochemical gradient 
of $\mathrm{H}^{+}$ions as they pass through complexes I (CI), II, III (CIII), and IV, activating the ATP synthase mechanism $[12,13]$.

During this oxidative processing, mitochondrial CI, a large enzymatic complex of over 40 subunits directly embedded in the inner mitochondrial membrane, plays a role as the dominant site where oxidative phosphorylation occurs [14]. Therefore, CI dysfunction in mitochondria has been considered an early cellular event leading to cell death by activating apoptotic signaling [15]. In this study, we investigated whether nobiletin regulated mitochondrial dysfunction that might specifically be mediated by the inhibition of CI or CIII, because they are the dominant sites that determine cellular metabolism and fate.

In the present study, we hypothesized that nobiletin might exert the regulatory effect to enhance antioxidant and metabolic functions of neuronal mitochondria under oxidative conditions, which were induced by the dysfunction of the ETC system, because it previously showed neuroprotective effects by regulating mitochondrial depolarization through $\mathrm{K}^{+}$channels [5]. Also, we investigated if the effects of nobiletin might be correlated with antiapoptotic signaling cascades in dissociated cortical neurons. For these purposes, we isolated pure mitochondria from rat cortical neurons, and pharmacologically induced the inhibition of CI and CIII.

\section{MATERIALS AND METHODS}

\section{Materials}

Dulbecco's modified Eagle’s medium (DMEM), Minimal Essential Medium (MEM), Neurobasal medium, fetal bovine serum (FBS), B-27 serum-free supplement, glutamine and penicillin/ streptomycin were purchased from Gibco BRL (Grand Island, NY, USA). Antibodies against AIF and TATA binding protein (TBP) were purchased from Santa Cruz Biotechnology Inc (Santa Cruz, CA, USA) and Abcam (Cambridge, UK), respectively. 3-(4,5-dimethylthiazol-2-yl)-2,5-diphenyl tetrazolium bromide (MTT) and 2,7'-dichlorofluorescin diacetate (DCF-DA) were purchased from Amresco (Solon, OH, USA) and Sigma-Aldrich (st. Louis, MO, USA), respectively. Nobiletin was isolated and purified from the peel of Citrus sunki Hort. ex Tanaka in the Department of Biology at Jeju National University, as described previously [16]. All other reagents were obtained from Sigma-Aldrich unless otherwise indicated.

\section{Primary culture of cortical neurons}

Primary cortical neurons were prepared from the cerebral cortices of 1-day-old SD rats. The cortices were isolated from the neonatal brains and transferred to plating medium containing MEM supplemented with $10 \%$ FBS, $25 \mathrm{mM}$ glucose, $1 \mathrm{mM}$ sodium pyruvate, $25 \mathrm{mM}$ glutamine, and $1 \%$ penicillin/streptomycin. Then, the isolated tissues were dissociated by trituration using pipettes and plated onto poly-L-lysine-coated 24-well plates at a density of $1 \times 10^{5} \mathrm{cells} /$ well. After six hours, plating medium was replaced with Neurobasal media supplemented with 2\% B-27, 50 mM glutamine and $1 \%$ penicillin/streptomycin, and half of the culture medium was replaced every four days. The neurons were incubated at $37^{\circ} \mathrm{C}$ in a humidified $5 \% \mathrm{CO}_{2} / 95 \% \mathrm{O}_{2}$ atmosphere and used in the experiments at DIV 7 8. All experiments and animal procedures were performed after approval from the Animal Care and Use Committee of Jeju National University.

\section{Culture of HT-22 neurons}

HT-22 neurons, an immortalized hippocampal cell line, were a generous gift from Dr. B.H. Lee (Medicine and Science, Gachon University, South Korea). The cells were cultured in DMEM supplemented with $10 \% \mathrm{FBS}$ and $1 \%$ penicillin/streptomycin and incubated at $37^{\circ} \mathrm{C}$ in a humidified $5 \% \mathrm{CO}_{2} / 95 \% \mathrm{O}_{2}$ atmosphere. The cells were the passage number 3 and used up to passage number 9 . The cultured cells were plated in $100 \mathrm{~mm}$ plates at a density $4 \times 10^{4} \mathrm{cells} /$ well, grown for 48 hours, and then used for the Western blotting experiments.

\section{Preparation of pure mitochondria from rat brain cortices}

Mitochondria were isolated from rat brain cortices, as previously described [17]. Briefly, the cortices were separated from the brains of 14 to 17-day-old rats and placed in EGTA-containing isolation buffer (IB) and homogenized in a Dounce-type tissue grinder (Kimble Chase). Pestles A and B were used sequentially with clearances of $0.0035 \sim 0.0065 \mathrm{~mm}$ and $0.0010 \sim 0.0030 \mathrm{~mm}$, respectively. The IB was containing (in $\mathrm{mM}$ ); 225 Mannitol, $75 \mathrm{Su}$ crose, 5 HEPES, 3 EGTA, $0.1 \%$ BSA, adjusted at pH 7.4 with $\mathrm{KOH}$ $(1 \mathrm{~N})$. The homogenates were centrifuged at $600 \times g$ for 10 minutes and then, the supernatant was transferred to a new tube and centrifuged again at $12,000 \times g$ for 10 minutes. The pellet containing mitochondria was resuspended in IB without EGTA and homogenized using Dounce-type tissue grinders. The clearances of pestle $\mathrm{A}$ and $\mathrm{B}$ at this stage were $0.0028 \sim 0.0047 \mathrm{~mm}$ and $0.0008 \sim 0.0022$ $\mathrm{mm}$, respectively. The homogenates were centrifuged again at $12,000 \times g$ for 10 minutes. All of the above procedures were conducted at $4^{\circ} \mathrm{C}$. The isolated mitochondrial proteins were quantified using Bio-Rad protein assay dye. The oxygen consumption rate (OCR) was measured, and electron microscopy (EM) was performed to evaluate the metabolic activity and morphology of the isolated mitochondria. 


\section{Measurements of mitochondrial viability and ROS in pure isolated mitochondria}

After isolating pure mitochondria from the rat brain cortices, the mitochondria suspension $(0,100,250,500,1,000 \mu \mathrm{g} / \mathrm{ml})$ was incubated in mitochondrial assay buffer for 10 minutes at $37^{\circ} \mathrm{C}$ with $0.4 \mathrm{mg} / \mathrm{ml}$ [3-(4,5-dimethylthiazol-2-yl)-2,5-diphenyl tetrazolium bromide] (MTT) and 2', 7'-dichlorofluorescin diacetate (DCF-DA, $25 \mu \mathrm{M}$ ) for measuring mitochondrial viability and ROS, respectively. 2', 7'-dichlorofluorescin (DCF) has been widely applied for monitoring ROS production in cells as well as isolated mitochondria and DCF-DA is commonly used for measuring calcium-induced ROS in isolated brain or liver mitochondria [13, 18-21]. MTT assay is useful to measure the viability of isolated mitochondria [22]. The mitochondrial assay buffer was containing (in mM); 220 mannitol, 70 sucrose, $10 \mathrm{KH}_{2} \mathrm{PO}_{4}, 5 \mathrm{MgCl}_{2}, 2$ HEPES, 1 EGTA and $0.2 \%$ fatty acid-free BSA (w/v) and $\mathrm{pH}$ was set at 7.2. The mitochondria loaded in the suspension were treated with nobiletin or other reagents in 96 well plates. The mitochondrial suspension was plated at $200 \mu \mathrm{l} /$ well $(100 \mu \mathrm{g} / \mathrm{ml}$ protein per well). Then, the fluorescence intensity of DCF-DA was measured using a fluorescence microplate reader (SpectraMax i3, Molecular devices Spectramax i3, Sunnyvale, CA, USA) at 485 and $535 \mathrm{~nm}$ excitation and emission wavelengths, respectively. The protein and mitochondrial viability were measured at an absorbance of $550 \mathrm{~nm}$ on a multifunctional plate reader and compared to nontreated control cells.

\section{Measurement of oxygen consumption rate (OCR) in pure isolated mitochondria}

The OCR of pure isolated mitochondria was measured using a Seahorse XF-24 extracellular flux analyzer (Seahorse Bioscience, Inc, North Billerica, MA, USA, belongs to 'Bio-Health Materials Core-Facility of Jeju National University') as according to the manufacturer's protocol. Briefly, $5 \mu \mathrm{g}$ of isolated mitochondria was suspended in $50 \mu \mathrm{l}$ assay medium and transferred to wells for OCR measurements. Mitochondrial assay medium was containing (in mM); 220 mannitol, 70 sucrose, $10 \mathrm{KH}_{2} \mathrm{PO}_{4}, 5 \mathrm{MgCl}_{2}, 2$ HEPES, 1 EGTA and $0.2 \%$ fatty acid-free BSA (w/v), and $\mathrm{pH}$ was set at 7.2. One day before the experiment, a sensor cartridge was placed into the calibration buffer and incubated overnight in a non- $\mathrm{CO}_{2}$ condition at $37^{\circ} \mathrm{C}$. The reagents listed below were added sequentially according to the manufacturer's protocol: ADP (1 $\mathrm{mM})$, oligomycin $(2 \mu \mathrm{g} / \mathrm{ml})$ as an inhibitor of mitochondrial ATP synthase, carbonyl cyanide 4-(trifluoromethoxy)phenylhydrazone (FCCP $2 \mu \mathrm{M})$ as an ETC system accelerator, and rotenone or antimycin $\mathrm{A}(0.5 \mu \mathrm{M})$ as CI and CIII inhibitors, respectively. The OCR data recorded by the sensor cartridges were analyzed using the
Seahorse XF-24 software according to the following formula. [Increment of OCR-linked ATP] $=[$ The last rate of OCR measured right before oligomycin addition]-[The minimal rate of OCR measured after oligomycin addition]

\section{Preparations of cytoplasmic and nuclear proteins}

The preparations of cytoplasmic and nuclear protein were performed using NE-PER nuclear and cytoplasmic extraction reagents (Invitrogen, Carlsbad, CA, USA) according to the manufacturer's protocol. For this experiment, HT-22 cells were seeded at a density of $1 \times 10^{6}$ cells/dish (100 mm diameter). The cells were treated with nobiletin and other reagents at the indicated time, washed twice, and collected in cold phosphate-buffered saline (PBS). After centrifuging (3,000 rpm), the cell pellets were resuspended in cytoplasmic extraction reagent. After centrifuging $(16,000 \times g)$ again, the supernatant cytoplasmic extract was transferred to a new tube and the nuclear pellets were resuspended in the nuclear extraction reagent, and centrifuged $(16,000 \times g)$. The supernatant nuclear protein extract was transferred to a new tube and stored at $-80^{\circ} \mathrm{C}$ until used.

\section{Western blotting analysis}

HT-22 cells were washed three times with PBS ( $\mathrm{pH} 7.4$ ) and lysed with modified RIPA buffer $(10 \mathrm{mM}$ TrisHCl, $150 \mathrm{mM} \mathrm{NaCl}$, $1 \mathrm{mM}$ EGTA, 0.1\% SDS, 1 mM NaF, 1 mM Na3VO4, 1 mM PMSF, $1 \mu \mathrm{g} / \mathrm{ml}$ aprotinin, and $1 \mu \mathrm{g} / \mathrm{ml}$ leupeptin, $\mathrm{pH}$ 7.4). The Protein (50 $\mu \mathrm{g}$ ) were separated by sodium dodecyl sulfate-polyacrylamide gel electrophoresis and then electrotransferred onto a polyvinylidene fluoride membrane (Bio-Rad Lab., Hercules, CA, USA) using Towbin transfer buffer (192 mM glycine, $25 \mathrm{mM}$ Tris, and 20\% methanol, $\mathrm{pH}$ 8.3). The blots were incubated with 5\% skim milk in TTBS (25 mM Tris, 150 mM NaCl, pH 7.4, containing 0.1\% Tween 20) for 1 hour at room temperature to block nonspecific binding. Subsequently, the membranes were incubated overnight at $4^{\circ} \mathrm{C}$ with anti-Nrf-2, anti-TBP, anti-HO- 1 , anti-AIF, and anti- $\beta$-actin antibodies. The blots were washed three times with TTBS and incubated with the appropriate horseradish peroxidase-conjugated secondary antibodies for 1 hour at room temperature. After several washes, the blots were developed using enhanced chemiluminescence detection reagents (Intron Biotechnology, Sungnam, Korea) according to the manufacturer's instructions. The optical densities of the bands were quantified with an Image J analyzer (http://rsb. info.nih.gov/ij/).

\section{Measurement of cell viability}

MTT was used to investigate the cytoprotective effect of nobiletin on cell viability. This protocol was based on the phenomenon 
that water-soluble MTT is converted to an insoluble purple formazan by mitochondria in living cells. Primary cortical neurons (DIV 4) were plated at a density of $15 \times 10^{4} \mathrm{cells} / \mathrm{ml}$ and $1 \mu \mathrm{M}$ arabinofuranosyl cytidine (Ara-C) was added to each culture well to block the growth of glial cells. Subsequently, the concentration of Ara-C was consistently kept at $1 \mu \mathrm{M}$ in the cell culture medium (4 days). After that, MTT solution $(0.4 \mathrm{mg} / \mathrm{ml})$ was added to the culture medium for 1 hour at $37^{\circ} \mathrm{C}$. Then, the supernatant was discarded, and the formazan was dissolved in dimethyl sulfoxide (DMSO). The absorbance was measured at $550 \mathrm{~nm}$ using a microplate reader (Model 550, Bio-Rad, USA).

\section{Mitochondrial respiratory complex I activity assay}

The activity of mitochondrial respiratory $\mathrm{CI}$ was determined using the MitoCheck Complex I Activity Assay Kit (Cayman Chemical Company ${ }^{\circledR}$, Ann Arbor, MI, USA), according to the manufacturer's instructions. To quantify the level of CI activity, $20 \mu \mathrm{g}$ of mitochondrial protein was used and the absorbance of all samples was measured at $340 \mathrm{~nm}$ for 15 minutes using a microplate reader (SpectraMax i3). The level of CI activity was quantified by the increase in absorbance per minute, and the experiment was conducted three times for the statistical analysis.

\section{Statistical analysis}

All data are presented as the mean \pm standard error of the mean (SEM), and the statistical analysis was performed by one- or twoway ANOVA using SPSS software. The differences between the groups were considered significant when the $\mathrm{p}$-value was $<0.05$ or 0.01 .

\section{RESULTS}

\section{ETC inhibition increases mitochondrial ROS}

The dysfunction of the mitochondrial ETC system in mammalian neurons, activates abnormal oxidative signaling cascades to excessively express ROS and then triggers pathogenic conditions such as stroke, ischemia, and most types of degenerative diseases [23-27]. To investigate the neuroprotective effects of nobiletin on mitochondrial dysfunction, we first induced the excessive production of ROS by inhibiting the ETC system in pure mitochondria $(500 \mu \mathrm{g} / \mathrm{ml})$ isolated from the brain cortices of 14 to 16-day-old SD rats. Rotenone or 6-OHDA, and antimycin A were used to inhibit CI and CIII, respectively, and their pharmacological effects were determined by measuring the level of ROS (Fig. 1). Dosedependence was tested by treating inhibitors to isolated mitochondria for 5 minutes (Fig. 1A, C, and E), and time-dependence was determined by treating $0.5 \mu \mathrm{M}$ rotenone, $5 \mu \mathrm{M}$ 6-OHDA, or
$0.5 \mu \mathrm{M}$ antimycin A (Fig. 1B, D, and E). Both CI inhibitors strongly increased mitochondrial ROS production in dose- and timedependent manners (Fig. 1A, ROT $0.5 \mu \mathrm{M}$ for $5 \mathrm{~min}=130.5 \pm 3.6 \%$, $\mathrm{n}=4, \mathrm{p}<0.01$ compared with the control; Fig. 1C, 6-OHDA $10 \mu \mathrm{M}$ for $5 \mathrm{~min}=177.7 \pm 5.4 \%, \mathrm{n}=4, \mathrm{p}<0.01$, compared with the control). Antimycin A, a CIII inhibitor, also significantly increased mitochondrial ROS production, as shown in Fig. $1 \mathrm{E}$ and $\mathrm{F}$.

\section{Nobiletin restores neuronal viability under CI inhibition}

Previously, nobiletin exhibited antioxidant effects against glutamate-induced toxicity via regulating mitochondrial cation channels [5]. Therefore, it was necessary to determine if nobiletin might have neuroprotective and antioxidant effects under mitochondrial dysfunction conditions, which induce ROS overexpression. Fig. 2 shows that, after loading with the DCF-DA fluorescence indicator for 10 minutes and then pretreating with nobiletin $(30 \mu \mathrm{M})$ for 5 minutes, the isolated mitochondria treated with rotenone $(0.5 \mu \mathrm{M}$, Fig. $2 \mathrm{~A}), 6$-OHDA (10 $\mu \mathrm{M}$, Fig. $2 \mathrm{~B})$ or antimycin A $(0.5 \mu \mathrm{M}$. Fig. 2C) for 5 minutes overexpressed ROS. Nobiletin showed an antioxidant effect to significantly reduce the ROS levels in mitochondria increased by rotenone or 6-OHDA (Fig. $2 \mathrm{~A}, \mathrm{ROT}=131.7 \pm 5.4 \%, \mathrm{n}=4, \mathrm{p}<0.01$ compared with no treatment; $\mathrm{ROT}+\mathrm{NOB}=101.0 \pm 2.1 \%, \mathrm{n}=6, \mathrm{p}<0.01$ compared with $\mathrm{ROT}$ alone; Fig. 2 B. $6-\mathrm{OHDA}=113.4 \pm 5.4 \%, \mathrm{n}=6, \mathrm{p}<0.01$, compared with no treatment; $6-\mathrm{OHDA}+\mathrm{NOB}=102.30 \pm 3.8 \%, \mathrm{n}=5, \mathrm{p}<0.05$ compared with the 6-OHDA alone). However, this antioxidant effect was not observed in the mitochondria in which antimycin-A excessively increased the ROS levels (Fig. $2 \mathrm{C}, \mathrm{AA}+\mathrm{NOB}=176 \pm 2.6 \%$, $\mathrm{n}=6$, not significant, compared with AA alone). These results indicate that the antioxidant effect of nobiletin might be involved in mitochondrial dysfunction mediated specifically by the inhibition of $\mathrm{CI}$ in the ETC system.

Next, we investigated whether the antioxidant effect of nobiletin specific to CI inhibition was also involved in the neuroprotective mechanism that preserved neuronal viability. Rotenone or antimycin A was added to the culture media of DIV-8 primary cortical neurons of rats for 24 hours with or without one-hour pretreatment with nobiletin $(30 \mu \mathrm{M})$, and neuronal viability was measured by the MTT assay. Without nobiletin pretreatment (ROT alone), rotenone significantly reduced neuronal viability. In contrast, nobiletin-pretreated neurons (ROT+ and $\mathrm{NOB}+$ ) showed viability similar to that of the non-treated neurons (Fig. $3 \mathrm{~A}, \mathrm{ROT}=70.5 \pm 1.4 \%, \mathrm{n}=6, \mathrm{p}<0.05$, compared with no treatment; $\mathrm{ROT}+\mathrm{NOB}=91.4 \pm 1.6 \%, \mathrm{n}=6, \mathrm{p}<0.01$ compared with ROT alone). However, pretreatment with nobiletin did not exert neuroprotective effects to restore the cell viability under CIII inhibition induced by antimycin $\mathrm{A}$ (Fig. $3 \mathrm{~B}, \mathrm{AA}=75.9 \pm 4.6 \%, \mathrm{n}=5, \mathrm{p}<0.01$, 
A

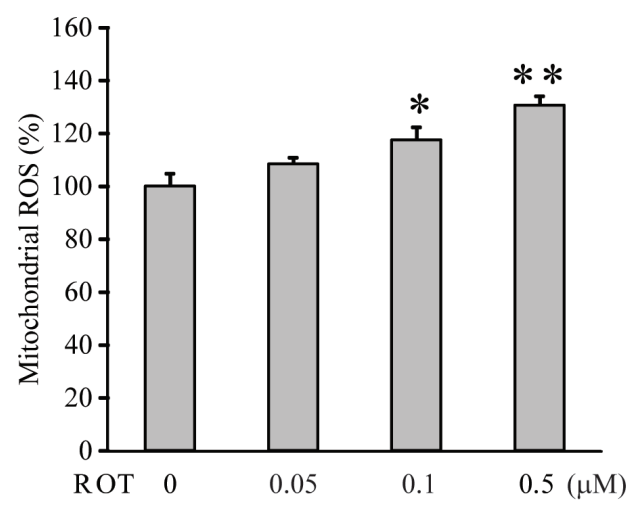

C

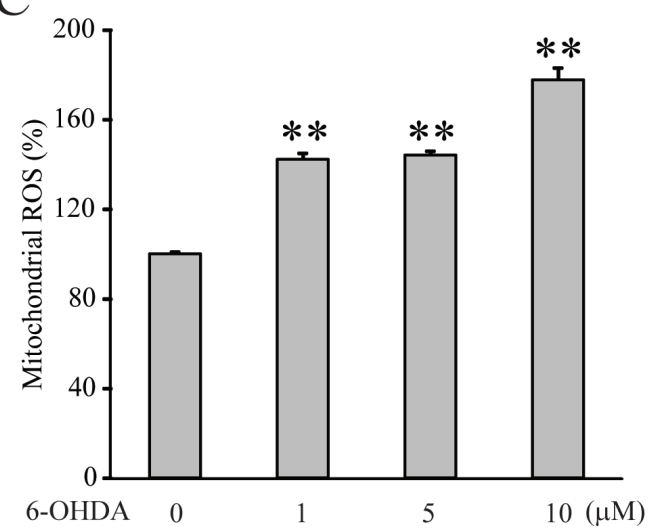

E

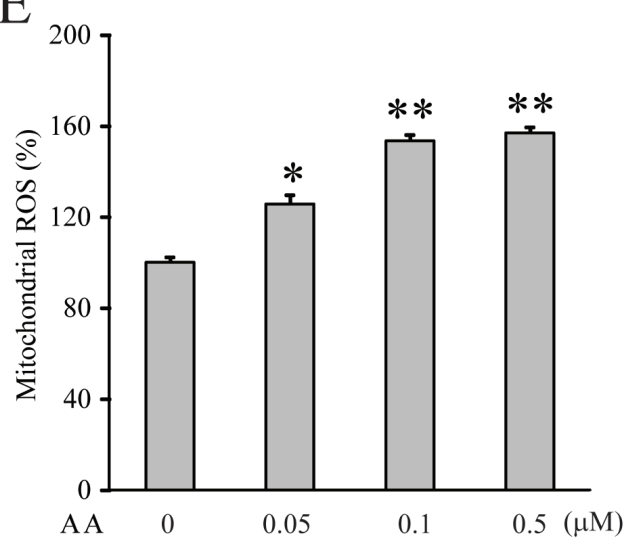

B

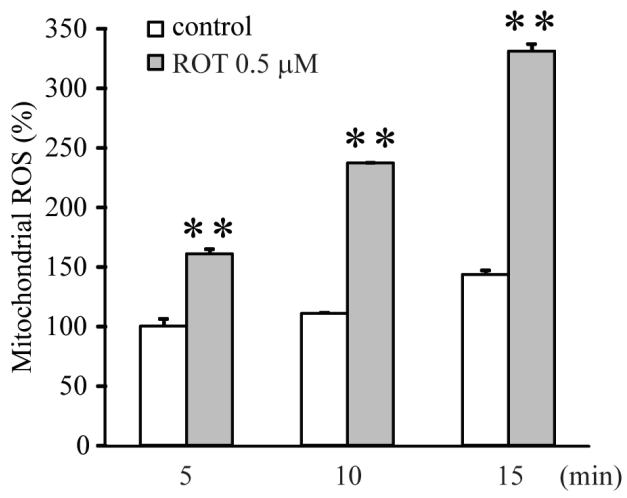

D

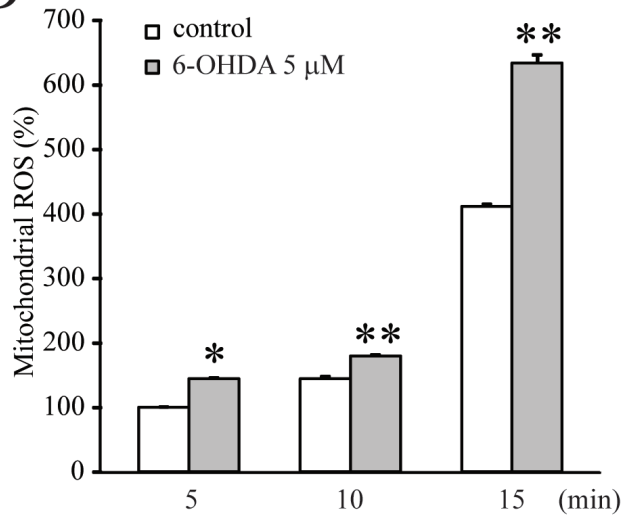

$\mathrm{F}$

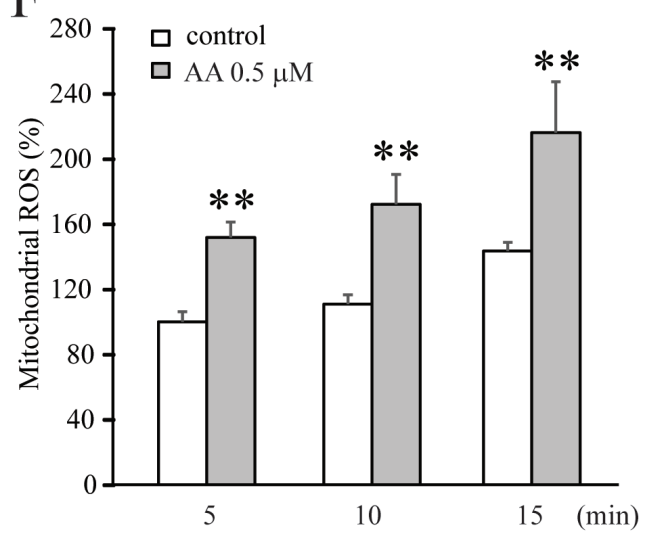

Fig. 1. The inhibition of ETC CI or CIII significantly increases the production of mitochondrial ROS. The mitochondrial sample (500 $\mu \mathrm{g} / \mathrm{ml})$ was loaded with DCF-DA $(25 \mu \mathrm{M})$ for 10 minutes, then each inhibitor was added for 5, 10 or 15 minutes. (A, C, E) Rotenone, 6-OHDA, or antimycin A at each concentration was treated to the isolated mitochondria for 5 minutes to test the dose-dependent effects on the ROS production. (B, D, F) $0.5 \mu \mathrm{M}$ rotenone, $5 \mu \mathrm{M}$ 6-OHDA, or $0.5 \mu \mathrm{M}$ antimycin A was treated during the indicated time for testing the time-dependence. All inhibitors dose- and timedependently increased ROS production in the isolated mitochondria. Data indicate the mean \pm S.E. ${ }^{*} \mathrm{p}<0.05$ and ${ }^{* *} \mathrm{p}<0.01$, compared with the control group.

compared with no treatment; $\mathrm{AA}+\mathrm{NOB}=76.2 \pm 0.8 \%, \mathrm{n}=6$, not significant, compared with AA alone).
Nobiletin enhances the OCR of mitochondria under CI inhibition

Because nobiletin seems to regulate mitochondria with an anti- 
A

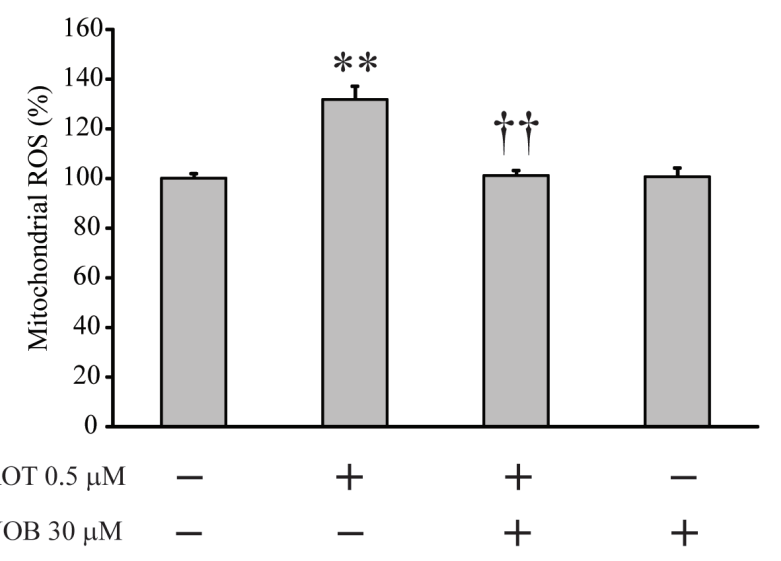

B

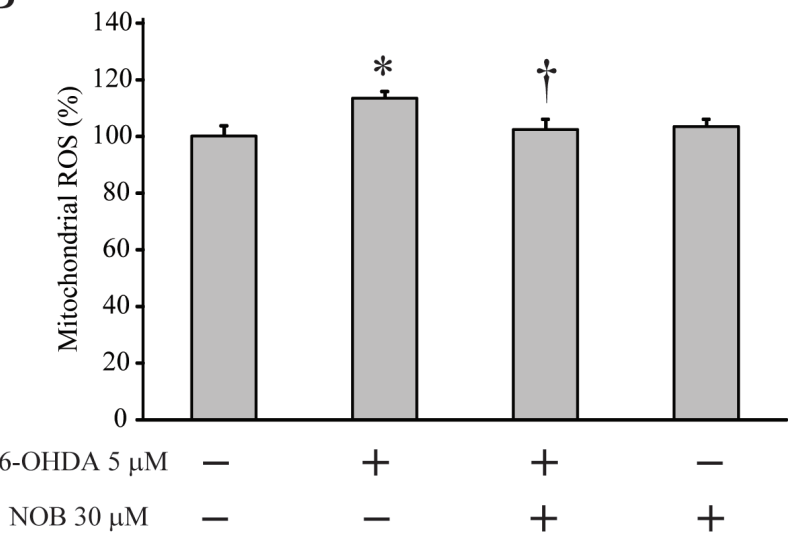

$\mathrm{C}$

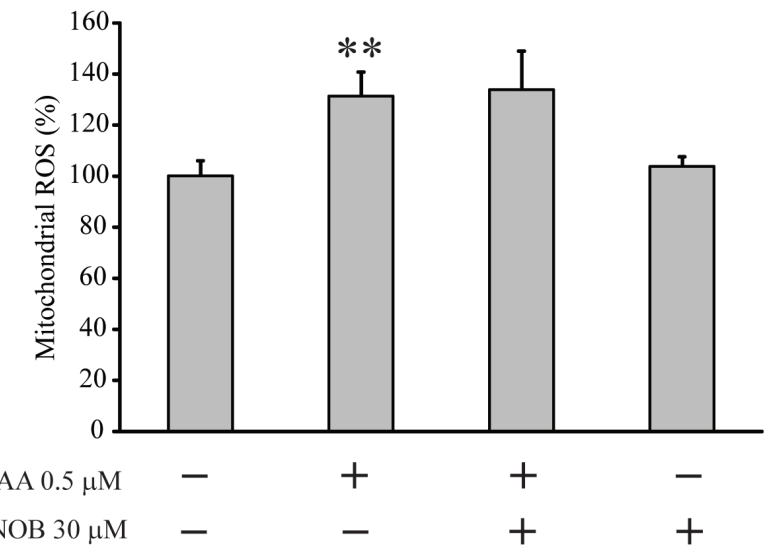

Fig. 2. Nobiletin effectively reduces the mitochondrial ROS under CI inhibition. Isolated mitochondria were pretreated with nobiletin (NOB, 30 $\mu \mathrm{M})$ for 5 minutes and then treated with each inhibitor of ETC complexes for 5 minutes. (A, B) Pretreatment with nobiletin completely blocked the effects of $\mathrm{CI}$ inhibitors to increase mitochondrial ROS (ROT+NOB, 6-OHDA+NOB). (C) The changes of mitochondrial ROS under CIII inhibition. Nobiletin did not exert the effect to reduce ROS level that was increased by treatment with antimycin $\mathrm{A}(\mathrm{AA}+\mathrm{NOB})$. Data indicate the mean \pm S.E. ${ }^{*} \mathrm{p}<0.05$ and ${ }^{* *} \mathrm{p}<0.01$, compared with the control group; ${ }^{\dagger} \mathrm{p}<0.05$ and ${ }^{\dagger \dagger} \mathrm{p}<0.01$, compared with each inhibitor alone. oxidant effect under CI inhibition, it was necessary to investigate whether it affected the metabolic function of mitochondria in neurons. The primary metabolic function of mitochondria is to produce ATP under physiological conditions, even though they can also act as calcium stores and ROS regulators. Figs. 4 and 5 show changes in the OCR in pure isolated mitochondria measured by the Mito Stress assay. From these OCR measurements, we calculated the ATP-linked OCR and coupling efficiency, which showed the coupling ratio of oxidation to phosphorylation. This is a well-known index indicating how much ATP generation is enhanced per each $\mathrm{O}_{2}$ uptake [28]. After injecting nobiletin, the OCR was slightly increased compared to the non-injected group, with no statistical significance. We also observed slight changes in ATPlinked OCR and coupling efficiency at a high concentration of nobiletin, but the changes were not significant (Fig. 4B, ATP-linked OCR, NOB, $1=346.3 \pm 24.4,10=360.2 \pm 33.7$, or $30 \mu \mathrm{M}=403.4 \pm 16.5$, $\mathrm{n}=5$; Fig. 4 C, NOB, 1, 10, or $30 \mu \mathrm{M}, 181.3 \pm 21.7,207.3 \pm 38.8$, $207.9 \pm 29.0, \mathrm{n}=5$, not significant). In contrast, OCR was positively influenced by nobiletin under the condition of $\mathrm{CI}$ inhibition. In this experiment, we first observed the effects of $\mathrm{CI}$ inhibition by removing CI substrates on OCR under a control condition (Fig. 5A). Then, nobiletin was tested by injecting after ADP injection under $\mathrm{CI}$ inhibition, because it was necessary to clearly identify the significance between conditions (Fig. 5B). Mitochondrial OCR was downregulated under the CI substrate-free condition, and both the OCR-linked ATP and coupling efficiency were significantly reduced (Fig. 5A, C and D, CI inhibition; OCR-linked ATP $=524.2 \pm 16.1, n=4, p<0.01$, compared with Control; Coupling Efficiency $=154.4 \pm 7.9, \mathrm{n}=4, \mathrm{p}<0.01$, compared with Control). In this experiment, the ATP-linked OCR that was reduced under CI substrate-free conditions was slightly but significantly restored by injection with nobiletin (Fig, 5B, C, CI inhibition+NOB; ATPlinked OCR $=573.1 \pm 9.9, \mathrm{n}=4, \mathrm{p}<0.05$, compared with $\mathrm{CI}$ inhibition). The increase in coupling efficiency was also significant in 'NOB+CI inhibition' (Fig. 5D, Coupling Efficiency $=171.7 \pm 1.3$, $\mathrm{n}=4, \mathrm{p}<0.05$ ). This strongly suggests that the regulatory effects of nobiletin on the oxidative metabolism were activated under the mitochondrial dysfunction caused by the inhibition of $\mathrm{CI}$ in the ETC system.

\section{Nobiletin enhances mitochondrial viability under active CI}

The results in Fig. 5 suggest the possibility that the nobiletinenhanced OCR resulted from an increase in mitochondrial viability rather than the upregulation of oxidative metabolism under $\mathrm{CI}$ inhibition. To confirm this, we investigated whether nobiletin directly affected the viability of isolated mitochondria. Nobiletin significantly increased mitochondrial viability under conditions 
A

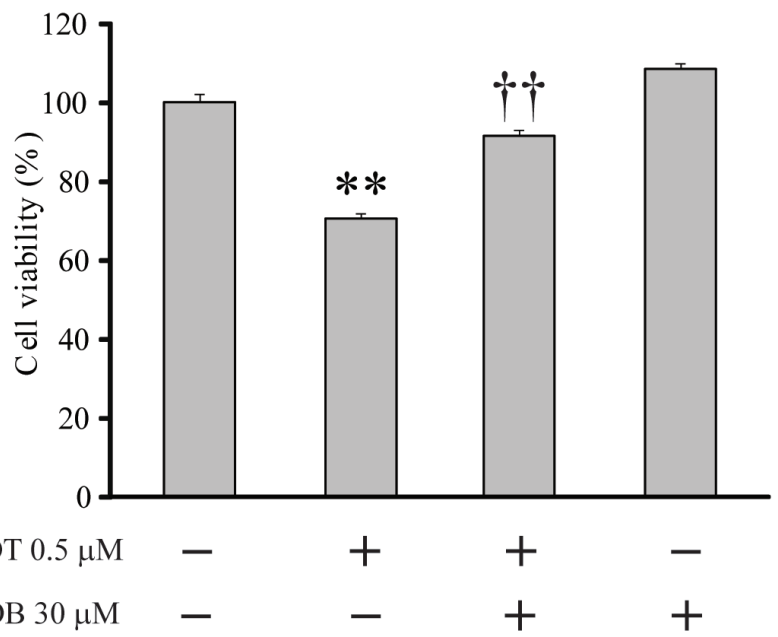

B

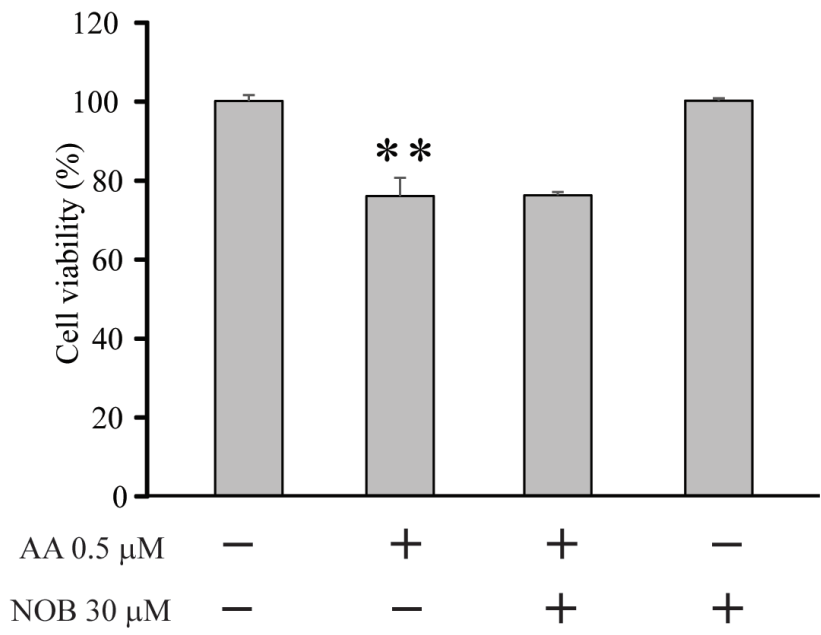

Fig. 3. Nobiletin effectively restores the viability of cortical neurons under CI inhibition. (A, B) The changes in viability of cortical neurons. Primary cortical neurons dissociated from rat brains were pretreated with (or without) nobiletin (NOB) for 1 hour and then incubated with rotenone (ROT, 0.5 $\mu \mathrm{M})$ or antimycin A (AA, $0.5 \mu \mathrm{M})$ for 24 hours. Neuronal viability was measured using an MTT assay. Nobiletin pretreatment effectively restored the neuronal viability under $\mathrm{CI}$ inhibition $\left(\mathrm{ROT}+, \mathrm{NOB}+\right.$ ) but not $\mathrm{CIII}$ inhibition $\left(\mathrm{AA}+, \mathrm{NOB}+\right.$ ). Data indicate the mean $\pm \mathrm{S} . \mathrm{E} .{ }^{*} \mathrm{p}<0.05$ and ${ }^{* *} \mathrm{p}<0.01, \mathrm{com}-$ pared with non-treated group; ${ }^{\dagger \dagger} \mathrm{p}<0.01$, compared with rotenone alone.

with active CI (Fig. 6A, Control, NOB, $1 \mu \mathrm{M}=126.8 \pm 0.7 \%, \mathrm{n}=6$, $\mathrm{p}<0.01$, compared with 'NOB 0'). However, this effect was not observed when CI was inhibited (CI inhibition, NOB, $1=82.7 \pm 1.1 \%$, $10=84.0 \pm 1.0 \%, 30 \mu \mathrm{M}=77.9 \pm 1.3 \%, \mathrm{n}=6)$, indicating that the improvement in the OCR by nobiletin under CI inhibition was attributed to metabolic upregulation, not due to changes in mitochondrial viability. We also investigated the effect of nobiletin on normal CI activity in isolated mitochondria using an ELISA assay (Fig. 6B). In this experiment, nobiletin significantly enhanced CI activity in a dose-dependent manner ( NOB $0.1,0.5,1,10$ or 30 $\mu \mathrm{M}, 100 \pm 0.8 \%, 109.5 \pm 0.7 \%, 110.1 \pm 0.7 \%, 177.1 \pm 1.4 \%, 219.7 \pm 1.5 \%$ and $253 \pm 1.0 \%, \mathrm{n}=7, \mathrm{p}<0.01$, compared with the control).

\section{Nobiletin inhibits the translocation of apoptosis-inducing factor}

Excessive mitochondrial ROS activates calpain, which translocates apoptosis-inducing factor (AIF) from the mitochondria, where AIF is localized under physiological conditions, to the nucleus [29,30]. Sequentially, nuclear AIF causes DNA fragmentation, resulting in apoptosis. Because the mitochondrial dysfunction associated with $\mathrm{CI}$ inhibition triggers the translocation of AIF, we investigated whether nobiletin exhibited an effect to block AIF translocation by Western blot analysis (Fig. 7) [31]. In HT-22 cells, pretreatment with rotenone significantly increased the expression level of AIF in both the nucleus (nAIF) and the cytosol (cAIF) compared to non-treated neurons (Fig. 7A, nAIF,
ROT $=146.2 \pm 8.7 \%, n=3, p<0.05$, compared with no treatment; Fig. $7 \mathrm{~B}, \mathrm{cAIF}, \mathrm{ROT}=168.5 \pm 6.5 \%, \mathrm{n}=3, \mathrm{p}<0.01$, compared with no treatment). However, this effect of rotenone on AIF expression was remarkably diminished under the presence of nobiletin, showing a similar AIF level as that in non-treated cells (Fig. 7A, nAIF, $\mathrm{ROT}+\mathrm{NOB}=86.0 \pm 9.4 \%, \mathrm{n}=3, \mathrm{p}<0.01$, compared with ROT alone; Fig. $7 \mathrm{~B}, \mathrm{cAIF}, \mathrm{ROT}+\mathrm{NOB}=131.0 \pm 6.0 \%, \mathrm{n}=3, \mathrm{p}<0.01$, compared with ROT alone).

According to the neuroprotective mechanisms, the activation of apoptotic signaling tended to accompany the activation of antioxidant signaling [32-35]. Furthermore, our results showing neuroprotective and anti-apoptotic effects suggest the possibility that nobiletin also has an effect to directly regulate the expression of antioxidant enzymes, such as $\mathrm{Nrf} 2$ or HO- 1 . Therefore, we investigated whether nobiletin influenced the expression of Nrf2 and HO- 1 in HT-22 cells (Fig. 8). The measurement of enzymes was performed under normal conditions because these enzymes are naturally overexpressed during the activation of apoptotic signaling [35-38]. In this experiment, treatment with nobiletin for 6 hours significantly and dose-dependently upregulated the expression of Nrf-2 and HO- 1 in HT-22 cells (Fig. 8B, Nrf-2, NOB $30 \mu \mathrm{M}=173.2 \pm 4.1 \%, \mathrm{n}=3, \mathrm{p}<0.01$, Fig. 8C, HO- 1 , NOB 30 $\mu \mathrm{M}=151.5 \pm 0.2 \%, \mathrm{n}=3, \mathrm{p}<0.01)$. 


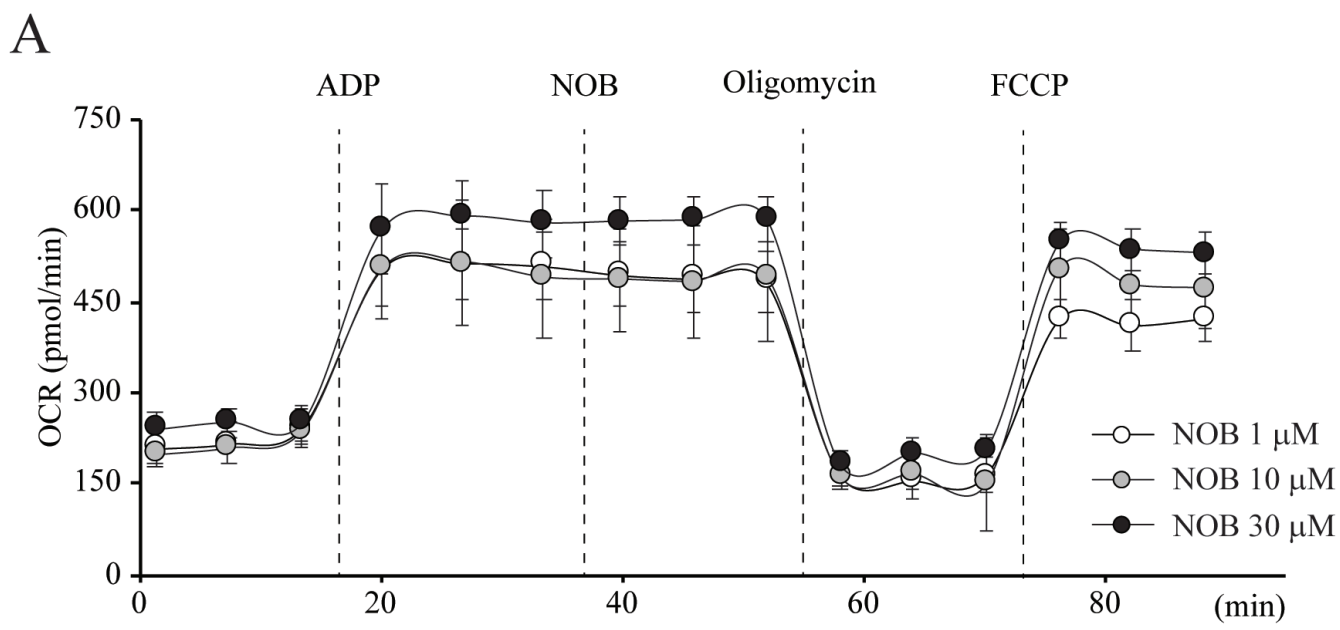

B

ATP - linked OCR

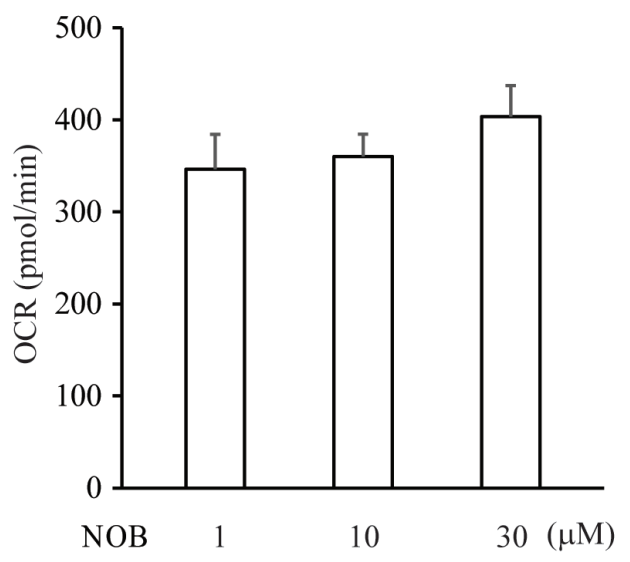

C

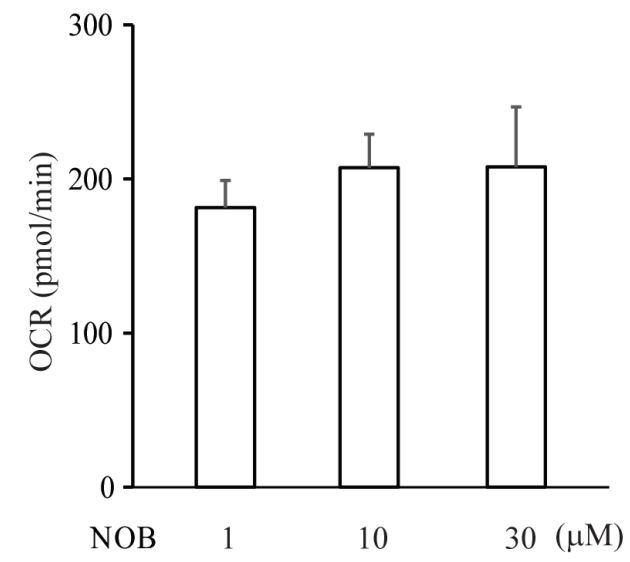

Fig. 4. Nobiletin does not affect the metabolic regulation of mitochondria under a normal condition. (A) The changes of OCR under each condition. The OCR of mitochondria was measured using a Seahorse XF-24 extracellular flux analyzer. ADP $(1 \mathrm{mM})$ was injected for enhancing OCR, oligomycin $(2 \mu \mathrm{g} / \mathrm{ml})$ as an inhibitor of mitochondrial ATP synthase, FCCP $(2 \mu \mathrm{M})$ as an ETC accelerator. The various concentrations of nobiletin were injected in pure isolated mitochondria under a normal condition and the changes of OCR were observed. (B, C) Averaged changes of ATP-linked OCR and coupling efficiency. Nobiletin treatment did not induce significant changes in mitochondrial OCR under a normal condition. Data indicate the mean \pm S.E.

\section{DISCUSSION}

Here, we report the neuroprotective and antioxidant effects of nobiletin under conditions of mitochondrial dysfunction particularly associated with the inhibition of CI in the ETC system. Its regulatory effects were observed in pure mitochondria isolated from the cortical neurons of rats, and the neuroprotective effects under CI inhibition were investigated in primary cortical neurons and HT-22 cells. The specificity of nobiletin against CI inhibition was confirmed by observing that 1 ) the downregulation of mitochondrial ROS production was seen only by treatment with rotenone or 6-OHDA (Fig. 2), and 2) the restoration of cell viabil- ity was observed only in cortical neurons treated with rotenone, not antimycin A (Fig. 3). These effects of nobiletin seemed to be associated with the activation of antioxidant and anti-apoptotic signaling (Fig. 7 and 8), upregulating the metabolic OCR of mitochondria under CI inhibition (Fig. 5).

The overproduction of mitochondrial ROS and alteration in mitochondrial redox homeostasis are involved in neuropathogenic and neurodegenerative diseases such as seizures, Alzheimer's, and Parkinson's diseases [39-44]. Particularly, CI and CIII of the ETC system are major sources of metabolic ATP production as well as ROS. CIII is the dominant site producing ROS in the circulatory and skeletal systems, whereas $\mathrm{CI}$ is responsible for most of 

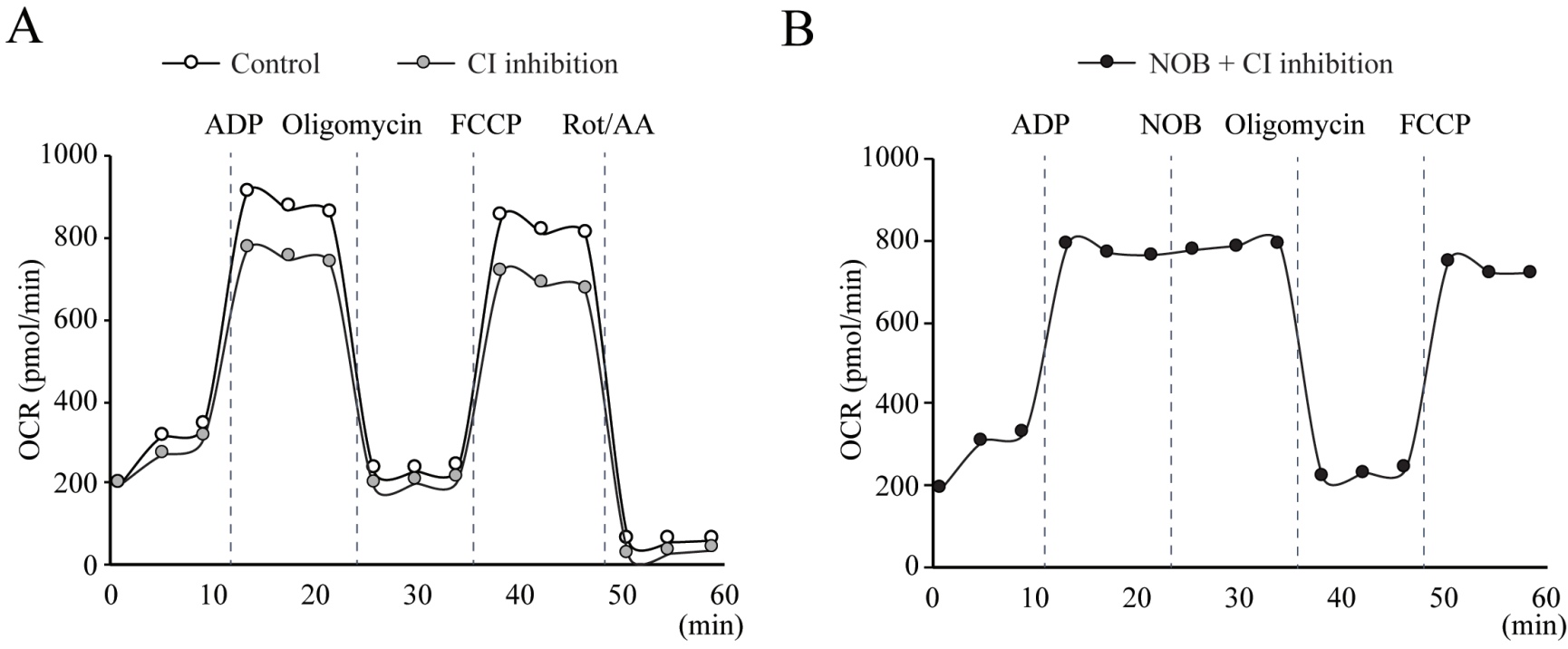

$\mathrm{C}$

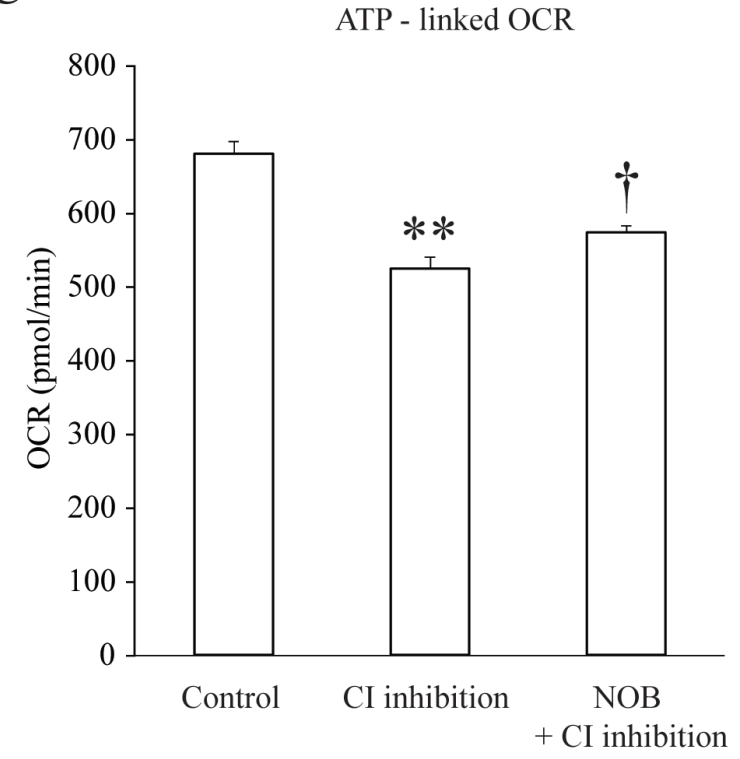

$\mathrm{D}$

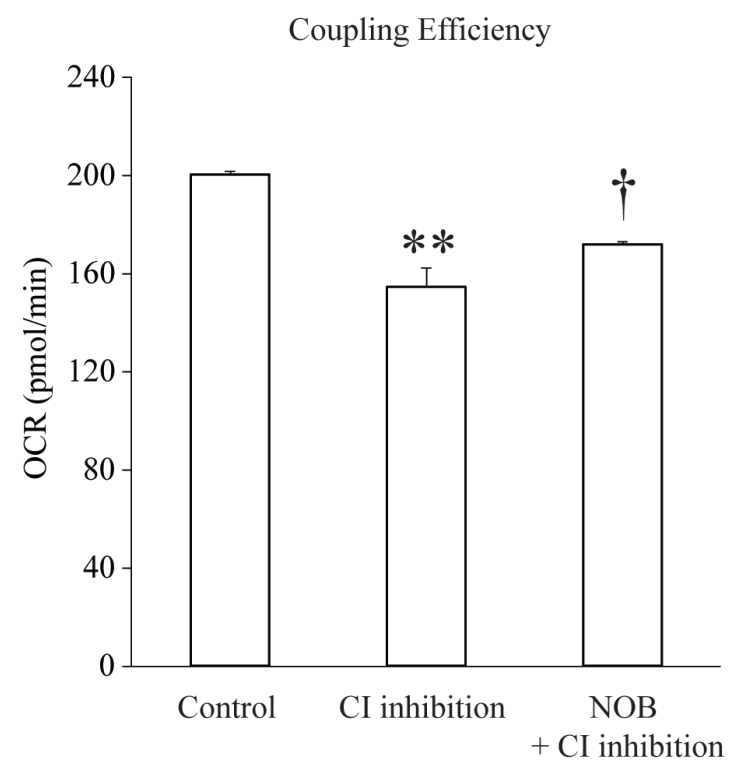

Fig. 5. Nobiletin partially but significantly restores the mitochondrial OCR under CI inhibition. (A) The changes of OCR levels. First, the effect of the absence of CI substrate (CI inhibition) on OCR was compared with the presence of CI substrate (Control). (B) To test the effect of nobiletin on OCR under $\mathrm{CI}$ inhibition, it was injected after ADP injection (NOB+CI inhibition). (C, D) The averaged values of OCR-linked ATP and coupling efficiency that were restored by nobiletin injection. The injection of nobiletin $(30 \mu \mathrm{M}, \mathrm{NOB}+\mathrm{CI}$ inhibition) slightly but significantly increased the level of OCRlinked ATP under the CI substrate free condition. Data indicate the mean \pm S.E. ${ }^{* *} \mathrm{p}<0.01$, compared with 'Control'; ${ }^{\dagger} \mathrm{p}<0.05$, compared with 'CI inhibition'.

the ROS produced in the nervous system [23, 45-47]. According to their cellular processes, CI generates ROS within the mitochondrial matrix, while CIII produces ROS in both the matrix and intermembrane space [48]. The downregulation of CI and/ or CIII, however, can trigger mitochondrial dysfunction, elevating the level of ROS [49-54]. Consistent with these reports, we also observed that the ROS level was significantly increased under either CI or CIII inhibition in this study. Interestingly, nobiletin effectively downregulated the level of mitochondrial ROS only under CI inhibition induced by rotenone or 6-OHDA in pure isolated mitochondria, whereas no effects were observed under CIII inhibition (Fig. 2A C). This means that nobiletin may activate a regulatory mechanism to target the oxidative signaling triggered by CI dysfunction in neuronal mitochondria. Furthermore, the CI inhibition-specific antioxidant effects of nobiletin seemed to contribute to the neuroprotective regulation in primary cortical neurons in which CI inhibition activated apoptotic signaling (Fig. $3 \mathrm{~A})$. The fact that nobiletin did not show neuroprotective effects 

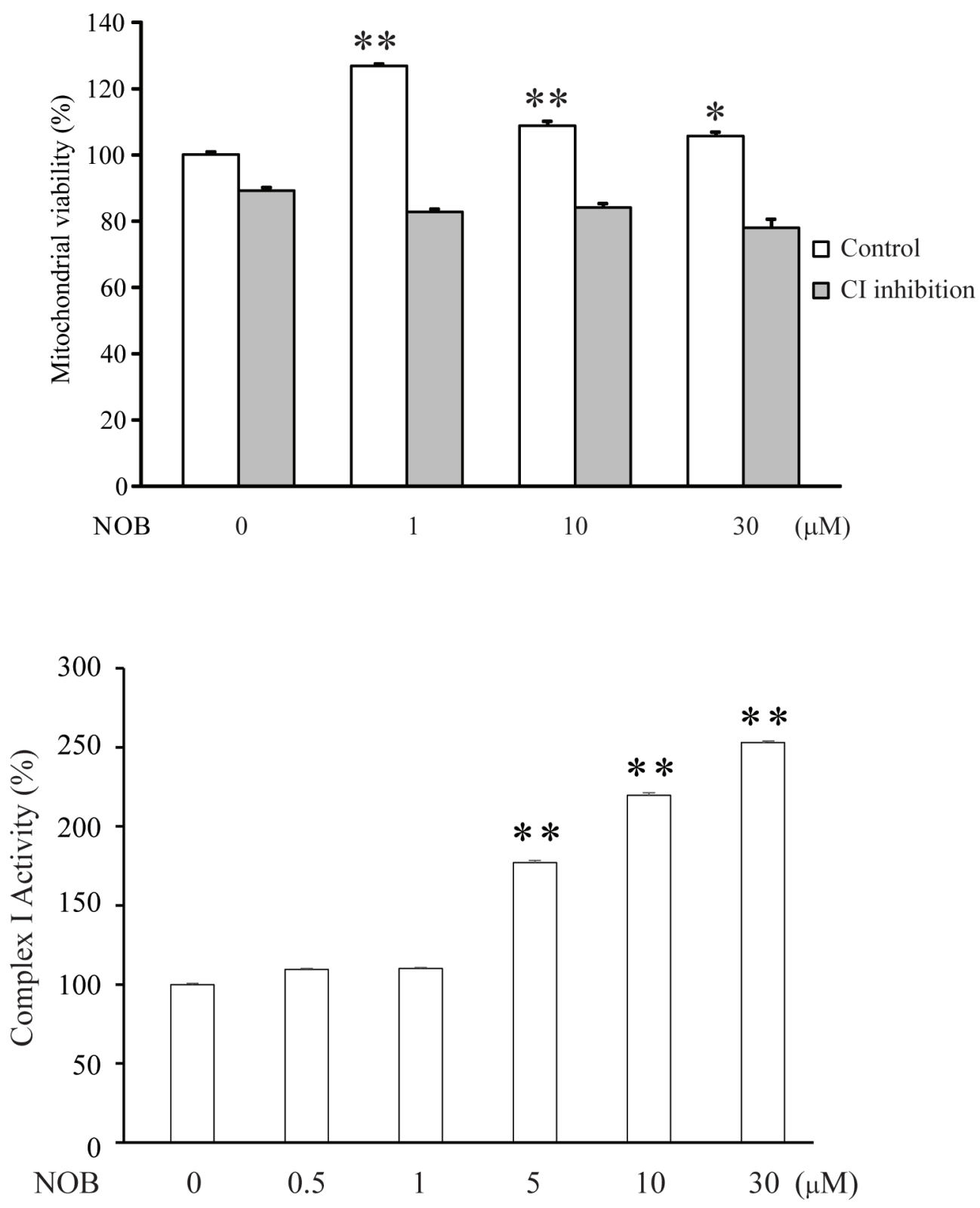

Fig. 6. Nobiletin enhances the mitochondrial viability and CI activity under a normal condition. (A) The changes of mitochondrial viability influenced by nobiletin with (CI inhibition) or without (Control) rotenone $(0.5 \mu \mathrm{M})$ treatment. Nobiletin significantly enhanced the mitochondrial viability only under a normal condition (Control). (B) The changes of CI activity that were dose-dependently enhanced by nobiletin. CI activity was determined by using MitoCheck Complex I Activity Assay Kit with $20 \mu \mathrm{g}$ of mitochondrial protein. The absorbance of all samples was measured at $340 \mathrm{~nm}$ for $15 \mathrm{~min}-$ utes. Data indicate the mean \pm S.E. ${ }^{*} \mathrm{p}<0.05$ and ${ }^{* *} \mathrm{p}<0.01$, compared with no treatment $(0 \mu \mathrm{M} \mathrm{NOB})$.

by treatment with antimycin $\mathrm{A}$ indicates the existence of regulatory functions to prevent mitochondrial dysfunction rather than simply eliminating ROS. Furthermore, without an increase in mitochondrial viability, nobiletin significantly enhanced the ATPlinked OCR, strongly suggesting that it may have modulatory effects to upregulate the metabolic functions of mitochondria under CI inhibition (Fig. 5 and 6A).

In this study, we did not address how nobiletin exerted neuro- protective effects on the mitochondrial dysfunction and apoptotic processes associated with the inhibition of CI of the ETC system, or what it was acting upon. In mitochondria, the electrons required for oxidative phosphorylation come from electric carriers such as nicotinamide adenine dinucleotide (NADH) and flavin adenine dinucleotide $\left(\mathrm{FADH}_{2}\right)$, which are activated in the tricarboxylic acid (TCA) cycle in the mitochondrial matrix space [55]. Here, CI plays a role in converting NAD from its reduced form, NADH, 
A
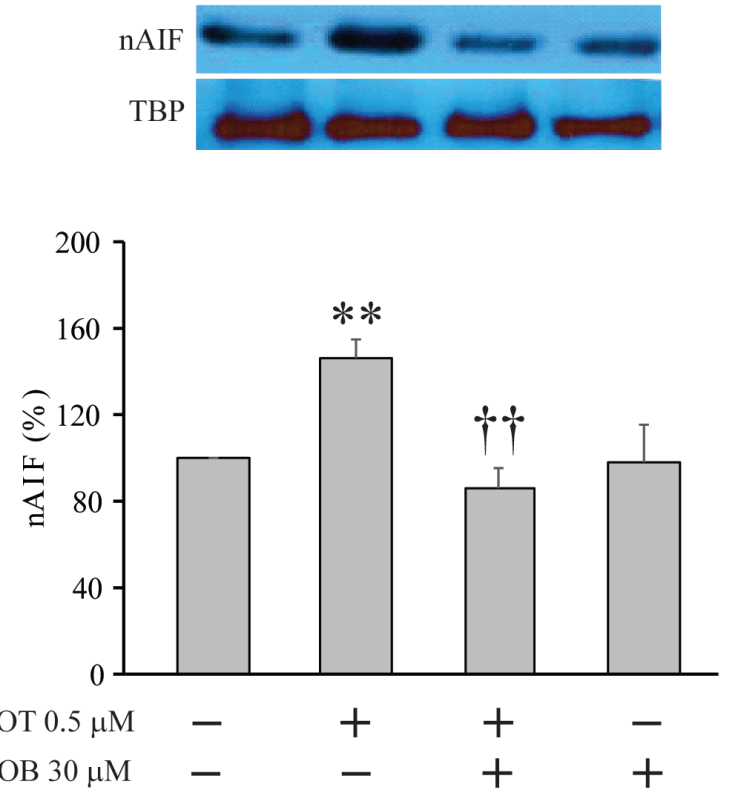

B
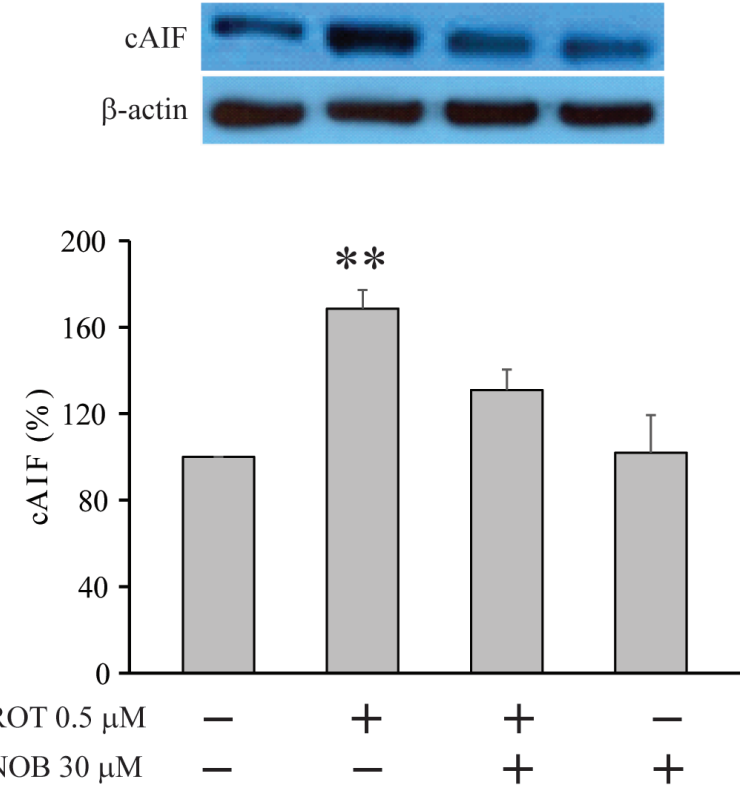

Fig. 7. Nobiletin effectively suppresses the translocation of AIF under CI inhibition. The changes of expression levels of AIFs in nucleus (A, nAIF) and cytosol (B, cAIF). The treatment with rotenone $(0.5 \mu \mathrm{M}, 6$ hours) significantly increased the expression of both nAIF and cAIF (ROT+). The pretreatment with nobiletin $(30 \mu \mathrm{M}, 1$ hour) significantly blocked the effect of rotenone, reducing the expression levels of AIFs (ROT+ and NOB+). Data indicate the mean \pm S.E. ${ }^{* *} \mathrm{p}<0.01$, compared with non-treated group; ${ }^{\dagger \dagger} \mathrm{p}<0.05$, compared with rotenone alone.

to its oxidized form, $\mathrm{NAD}^{+}$, and then synthesizing ATP. Nobiletin was previously reported to recover the reduced ATP metabolism during hypothyroidism in hippocampal mitochondria and activate mitochondrial substrate phosphorylation, indicating that nobiletin may directly or indirectly regulate CI of the ETC system [6]. Unexpectedly, we did not observe the effect of nobiletin to increase mitochondrial viability under CI inhibition (Fig. 6A), while its antioxidant effect to restore neuronal viability was exhibited under CI inhibition (Fig. 2 and 3). This may be due to an incomplete condition of isolated mitochondria which is totally different from the intact cellular condition. The restoration or prevention of mitochondrial damage requires a variety of signaling processes in the cellular level, including the crosstalk between mitochondria and nucleus [56].

Mitochondrial dysfunction induces the release of AIF from the mitochondria to the cytosol and its translocation to the nucleus induces DNA fragmentation and activates apoptotic signaling $[57,58]$. Consistent with these studies, CI inhibition by rotenone significantly augmented the expression level of AIF in both the cytosol and nucleus of HT-22 neurons, and nobiletin effectively blocked the rotenone-increased expression of AIF in this study (Fig. 7). This provides evidence for the presence of its regulatory function against apoptotic signaling under mitochondrial dys- function. Then, how does nobiletin downregulate apoptotic signaling? One possible mechanism is the involvement of antioxidant enzymes, which are overexpressed under oxidative conditions. Particularly, Nrf2 tends to be highly expressed to prevent cell damage or death in neurons that locate in the pathogenic region, showing active apoptotic signaling [58]. In this study, we confirmed that nobiletin directly enhanced the expression of $\mathrm{Nrf} 2$ as well as $\mathrm{HO}-1$ in HT-22 cells, even under normal conditions without any oxidative stimulation or mitochondrial dysfunction (Fig. 8). Together with previous studies, our results indicate that the neuroprotective effects of nobiletin under mitochondrial dysfunction may be associated with its function to activate antioxidant signaling cascades.

In the present study, we confirmed the neuroprotective and antioxidant effects of nobiletin against mitochondrial dysfunction, which was specifically mediated by CI inhibition of the ETC system. In addition to its antioxidant effects, nobiletin to regulate the metabolic functions of mitochondria under ETC dysfunction may be meaningful in Alzheimer's and Parkinson's diseases because the dysregulation of mitochondrial metabolism results in neuronal degeneration of the brain. Although further studies should address how the effects of nobiletin are specific to CI inhibition, our findings suggest the possibility that nobiletin has therapeutic potential in treating oxidative neurological and neurodegenerative diseases 
A

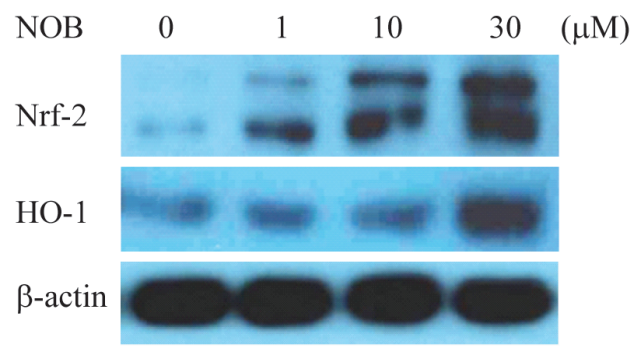

B

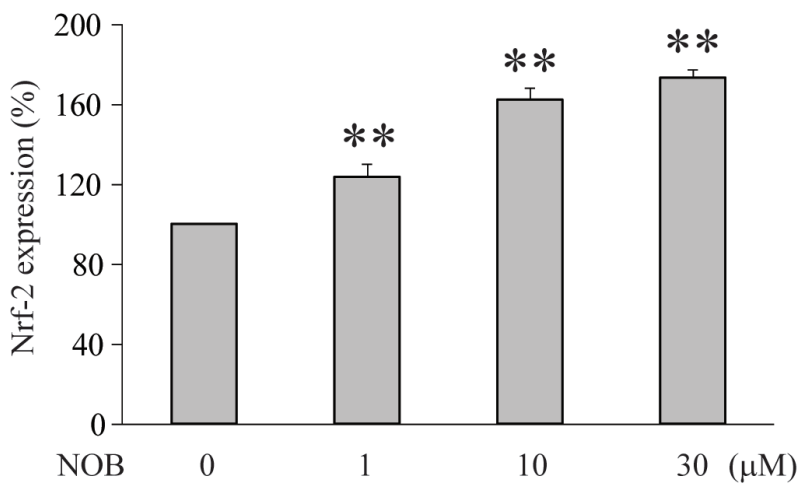

C

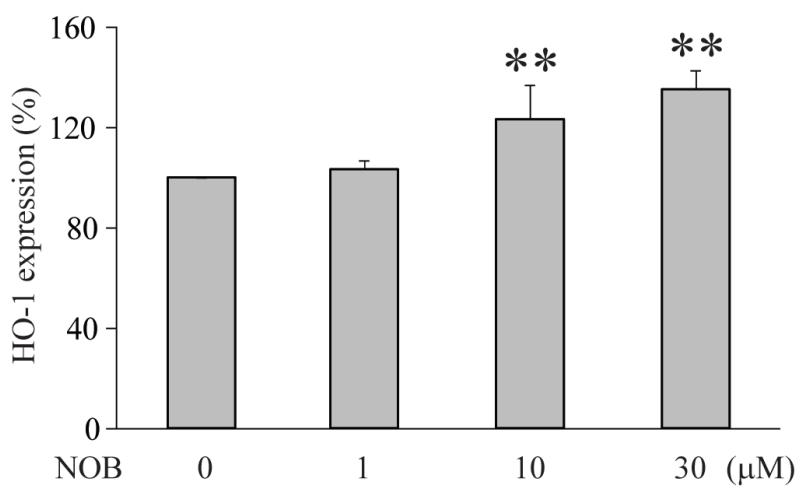

Fig. 8. Nobiletin dose-dependently increases the expression of antioxidant enzymes. (A) The changes of expression levels of Nrf-2 and HO-1 measured in HT-22 cells by a Western blot analysis. (B) The averaged expression level of Nrf-2. (C) The averaged expression level of HO-1. Both Nrf-2 and HO- 1 were significantly increased by nobiletin ( 6 hours treatment) in a dose-dependent manner under a normal condition. Data indicate the mean \pm S.E. ${ }^{* *} \mathrm{p}<0.01$, compared with ' $0 \mu \mathrm{M}$ NOB'.

mediated by mitochondrial dysfunction.

\section{ACKNOWLEDGEMENTS}

This work was supported by a research grant from the Jeju National University Hospital Research Fund of Jeju National University in 2019.

\section{REFERENCES}

1. Huang H, Li L, Shi W, Liu H, Yang J, Yuan X, Wu L (2016) The multifunctional effects of nobiletin and its metabolites in vivo and in vitro. Evid Based Complement Alternat Med 2016:2918796.

2. Onozuka H, Nakajima A, Matsuzaki K, Shin RW, Ogino K, Saigusa D, Tetsu N, Yokosuka A, Sashida Y, Mimaki Y, Yamakuni T, Ohizumi Y (2008) Nobiletin, a citrus flavonoid, improves memory impairment and Abeta pathology in a transgenic mouse model of Alzheimer's disease. J Pharmacol Exp Ther 326:739-744.

3. Yabuki Y, Ohizumi Y, Yokosuka A, Mimaki Y, Fukunaga K (2014) Nobiletin treatment improves motor and cognitive deficits seen in MPTP-induced Parkinson model mice. Neuroscience 259:126-141.

4. Yamamoto Y, Shioda N, Han F, Moriguchi S, Nakajima A, Yokosuka A, Mimaki Y, Sashida Y, Yamakuni T, Ohizumi Y, Fukunaga K (2009) Nobiletin improves brain ischemiainduced learning and memory deficits through stimulation of CaMKII and CREB phosphorylation. Brain Res 1295:218229.

5. Lee JH, Amarsanaa K, Wu J, Jeon SC, Cui Y, Jung SC, Park DB, Kim SJ, Han SH, Kim HW, Rhyu IJ, Eun SY (2018) Nobiletin attenuates neurotoxic mitochondrial calcium overload through $\mathrm{K}^{+}$influx and $\Delta \Psi_{\mathrm{m}}$ across mitochondrial inner membrane. Korean J Physiol Pharmacol 22:311-319.

6. Jojua N, Sharikadze N, Zhuravliova E, Zaalishvili E, Mikeladze DG (2015) Nobiletin restores impaired hippocampal mitochondrial bioenergetics in hypothyroidism through activation of matrix substrate-level phosphorylation. Nutr Neurosci 18:225-231.

7. Czabotar PE, Lessene G, Strasser A, Adams JM (2014) Control of apoptosis by the BCL-2 protein family: implications for physiology and therapy. Nat Rev Mol Cell Biol 15:49-63.

8. Giampazolias E, Tait SW (2016) Mitochondria and the hallmarks of cancer. FEBS J 283:803-814.

9. Segawa K, Kurata S, Yanagihashi Y, Brummelkamp TR, Matsuda F, Nagata S (2014) Caspase-mediated cleavage of phospholipid flippase for apoptotic phosphatidylserine exposure. Science 344:1164-1168.

10. Smaili SS, Hsu YT, Carvalho AC, Rosenstock TR, Sharpe JC, Youle RJ (2003) Mitochondria, calcium and pro-apoptotic proteins as mediators in cell death signaling. Braz J Med Biol Res 36:183-190.

11. Wei MC, Zong WX, Cheng EH, Lindsten T, Panoutsakopoulou V, Ross AJ, Roth KA, MacGregor GR, Thompson CB, 
Korsmeyer SJ (2001) Proapoptotic BAX and BAK: a requisite gateway to mitochondrial dysfunction and death. Science 292:727-730.

12. Dallner G, Sindelar PJ (2000) Regulation of ubiquinone metabolism. Free Radic Biol Med 29:285-294.

13. García-Ruiz C, Colell A, Marí M, Morales A, Fernández-Checa JC (1997) Direct effect of ceramide on the mitochondrial electron transport chain leads to generation of reactive oxygen species. Role of mitochondrial glutathione. J Biol Chem 272:11369-11377.

14. Gonzalez-Halphen D, Ghelli A, Iommarini L, Carelli V, Esposti MD (2011) Mitochondrial complex I and cell death: a semi-automatic shotgun model. Cell Death Dis 2:e222.

15. Bhatti JS, Bhatti GK, Reddy PH (2017) Mitochondrial dysfunction and oxidative stress in metabolic disorders - a step towards mitochondria based therapeutic strategies. Biochim Biophys Acta Mol Basis Dis 1863:1066-1077.

16. Choi SY, Hwang JH, Ko HC, Park JG, Kim SJ (2007) Nobiletin from citrus fruit peel inhibits the DNA-binding activity of NF-kappaB and ROS production in LPS-activated RAW 264.7 cells. J Ethnopharmacol 113:149-155.

17. Iglesias-González J, Sánchez-Iglesias S, Beiras-Iglesias A, SotoOtero R, Méndez-Álvarez E (2013) A simple method for isolating rat brain mitochondria with high metabolic activity: effects of EDTA and EGTA. J Neurosci Methods 213:39-42.

18. Puntel RL, Roos DH, Folmer V, Nogueira CW, Galina A, Aschner M, Rocha JB (2010) Mitochondrial dysfunction induced by different organochalchogens is mediated by thiol oxidation and is not dependent of the classical mitochondrial permeability transition pore opening. Toxicol Sci 117:133143.

19. Akopova OV, Kolchinskaya L, Nosar V (2017) The use of dichlorofluorescein as a probe for monitoring the effects of calcium on ROS production in mitochondria. Biochem Anal Biochem 6:3.

20. Tominaga H, Katoh H, Odagiri K, Takeuchi Y, Kawashima H, Saotome M, Urushida T, Satoh H, Hayashi H (2008) Different effects of palmitoyl-L-carnitine and palmitoyl-CoA on mitochondrial function in rat ventricular myocytes. Am J Physiol Heart Circ Physiol 295:H105-H112.

21. Kuka S, Tatarkova Z, Racay P, Lehotsky J, Dobrota D, Kaplan P (2013) Effect of aging on formation of reactive oxygen species by mitochondria of rat heart. Gen Physiol Biophys 32:415420.

22. Rezaiean Mehrabadi A, Jamshidzadeh A, Rashedinia M, Niknahad H (2015) Study of the effects of ATP suppliers and thiol reductants on toxicity of pioglitazone in isolated rat liver mitochondria. Iran J Pharm Res 14:825-832.

23. Barja G, Herrero A (1998) Localization at complex I and mechanism of the higher free radical production of brain nonsynaptic mitochondria in the short-lived rat than in the longevous pigeon. J Bioenerg Biomembr 30:235-243.

24. Kaminskyy VO, Zhivotovsky B (2014) Free radicals in cross talk between autophagy and apoptosis. Antioxid Redox Signal 21:86-102.

25. Circu ML, Aw TY (2010) Reactive oxygen species, cellular redox systems, and apoptosis. Free Radic Biol Med 48:749-762.

26. Ghezzi P, Jaquet V, Marcucci F, Schmidt HHHW (2017) The oxidative stress theory of disease: levels of evidence and epistemological aspects. Br J Pharmacol 174:1784-1796.

27. Kehrer JP, Klotz LO (2015) Free radicals and related reactive species as mediators of tissue injury and disease: implications for health. Crit Rev Toxicol 45:765-798.

28. Conley KE (2016) Mitochondria to motion: optimizing oxidative phosphorylation to improve exercise performance. J Exp Biol 219(Pt 2):243-249.

29. Bano D, Prehn JHM (2018) Apoptosis-inducing factor (AIF) in physiology and disease: the tale of a repented natural born killer. EBioMedicine 30:29-37.

30. Sevrioukova IF (2011) Apoptosis-inducing factor: structure, function, and redox regulation. Antioxid Redox Signal 14:2545-2579.

31. Li H, Chen G, Ma W, Li PA (2014) Water-soluble coenzyme q10 inhibits nuclear translocation of apoptosis inducing factor and cell death caused by mitochondrial complex I inhibition. Int J Mol Sci 15:13388-133400.

32. Nguyen T, Nioi P, Pickett CB (2009) The Nrf2-antioxidant response element signaling pathway and its activation by oxidative stress. J Biol Chem 284:13291-13295.

33. Khan MN, Ahmad I, Ansari MY, Haqqi TM (2017) Nrf2 inhibits apoptosis and suppresses oxidative stress by activating NOX4/ERK1/2/ELK1 signaling axis in human chondrocytes. Arthritis Rheumatol 69(Suppl 10):55.

34. Khan NM, Ahmad I, Haqqi TM (2018) Nrf2/ARE pathway attenuates oxidative and apoptotic response in human osteoarthritis chondrocytes by activating ERK1/2/ELK1-P70S6KP90RSK signaling axis. Free Radic Biol Med 116:159-171.

35. Paladino S, Conte A, Caggiano R, Pierantoni GM, Faraonio R (2018) Nrf2 pathway in age-related neurological disorders: insights into microRNAs. Cell Physiol Biochem 47:19511976.

36. He X, Lin GX, Chen MG, Zhang JX, Ma Q (2007) Protection against chromium (VI)-induced oxidative stress and apoptosis by Nrf2. Recruiting Nrf2 into the nucleus and disrupting 
the nuclear Nrf2/Keap1 association. Toxicol Sci 98:298-309.

37. Kim JW, Li MH, Jang JH, Na HK, Song NY, Lee C, Johnson JA, Surh YJ (2008) 15-Deoxy-Delta(12,14)-prostaglandin J(2) rescues $\mathrm{PC} 12$ cells from $\mathrm{H} 2 \mathrm{O} 2$-induced apoptosis through Nrf2-mediated upregulation of heme oxygenase-1: potential roles of Akt and ERK1/2. Biochem Pharmacol 76:1577-1589.

38. Jeong JY, Cha HJ, Choi EO, Kim CH, Kim GY, Yoo YH, Hwang HJ, Park HT, Yoon HM, Choi YH (2019) Activation of the Nrf2/HO-1 signaling pathway contributes to the protective effects of baicalein against oxidative stress-induced DNA damage and apoptosis in HEI193 Schwann cells. Int J Med Sci 16:145-155.

39. Alzheimer's Association (2016) 2016 Alzheimer's disease facts and figures. Alzheimers Dement 12:459-509.

40. Grimm A, Friedland K, Eckert A (2016) Mitochondrial dysfunction: the missing link between aging and sporadic Alzheimer's disease. Biogerontology 17:281-296.

41. Ibáñez P, Bonnet AM, Débarges B, Lohmann E, Tison F, Pollak P, Agid Y, Dürr A, Brice A (2004) Causal relation between alpha-synuclein gene duplication and familial Parkinson's disease. Lancet 364:1169-1171.

42. Indo HP, Yen HC, Nakanishi I, Matsumoto K, Tamura M, Nagano Y, Matsui H, Gusev O, Cornette R, Okuda T, Minamiyama Y, Ichikawa H, Suenaga S, Oki M, Sato T, Ozawa T, Clair DK, Majima HJ (2015) A mitochondrial superoxide theory for oxidative stress diseases and aging. J Clin Biochem Nutr 56:1-7.

43. Kishida KT, Klann E (2007) Sources and targets of reactive oxygen species in synaptic plasticity and memory. Antioxid Redox Signal 9:233-244.

44. Rego AC, Oliveira CR (2003) Mitochondrial dysfunction and reactive oxygen species in excitotoxicity and apoptosis: implications for the pathogenesis of neurodegenerative diseases. Neurochem Res 28:1563-1574.

45. Turrens JF, Alexandre A, Lehninger AL (1985) Ubisemiquinone is the electron donor for superoxide formation by complex III of heart mitochondria. Arch Biochem Biophys 237:408-414.

46. Muller FL, Liu Y, Van Remmen H (2004) Complex III releases superoxide to both sides of the inner mitochondrial mem- brane. J Biol Chem 279:49064-49073.

47. Fato R, Bergamini C, Leoni S, Strocchi P, Lenaz G (2008) Generation of reactive oxygen species by mitochondrial complex I: implications in neurodegeneration. Neurochem Res 33:2487-2501.

48. Brand MD (2010) The sites and topology of mitochondrial superoxide production. Exp Gerontol 45:466-472.

49. Guzy RD, Hoyos B, Robin E, Chen H, Liu L, Mansfield KD, Simon MC, Hammerling U, Schumacker PT (2005) Mitochondrial complex III is required for hypoxia-induced ROS production and cellular oxygen sensing. Cell Metab 1:401408.

50. Greenamyre JT, Sherer TB, Betarbet R, Panov AV (2001) Complex I and Parkinson's disease. IUBMB Life 52:135-141.

51. Lenaz G (2001) The mitochondrial production of reactive oxygen species: mechanisms and implications in human pathology. IUBMB Life 52:159-164.

52. Murphy AN, Fiskum G, Beal MF (1999) Mitochondria in neurodegeneration: bioenergetic function in cell life and death. J Cereb Blood Flow Metab 19:231-245.

53. Piskernik C, Haindl S, Behling T, Gerald Z, Kehrer I, Redl H, Kozlov AV (2008) Antimycin A and lipopolysaccharide cause the leakage of superoxide radicals from rat liver mitochondria. Biochim Biophys Acta 1782:280-285.

54. Turrens JF (1997) Superoxide production by the mitochondrial respiratory chain. Biosci Rep 17:3-8.

55. Martínez-Reyes I, Chandel NS (2020) Mitochondrial TCA cycle metabolites control physiology and disease. Nat Commun 11:102.

56. Saki M, Prakash A (2017) DNA damage related crosstalk between the nucleus and mitochondria. Free Radic Biol Med 107:216-227.

57. Veto S, Acs P, Bauer J, Lassmann H, Berente Z, Setalo G Jr, Borgulya G, Sumegi B, Komoly S, Gallyas F Jr, Illes Z (2010) Inhibiting poly(ADP-ribose) polymerase: a potential therapy against oligodendrocyte death. Brain 133(Pt 3):822-834.

58. Licht-Mayer S, Wimmer I, Traffehn S, Metz I, Brück W, Bauer J, Bradl M, Lassmann H (2015) Cell type-specific Nrf2 expression in multiple sclerosis lesions. Acta Neuropathol 130:263277. 